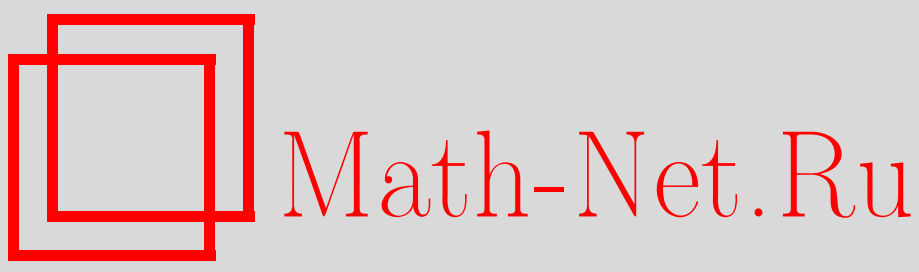

И. В. Аржанцев, О действиях редуктивных групп с однопараметрическим семейством сферических орбит, Матем. сб., 1997, том 188, номер 5, 3-20

DOI: https://doi.org/10.4213/sm222

Использование Общероссийского математического портала Math-Net.Ru подразумевает, что вы прочитали и согласны с пользовательским соглашением

http: //www. mathnet.ru/rus/agreement

Параметры загрузки:

IP : 54.196 .121 .252

26 апреля 2023 г., 13:04:28 
УДК 512.74

\author{
И.В. Аржанцев
}

\title{
О действиях редуктивных групп с однопараметрическим семейством сферических орбит
}

\begin{abstract}
Данная работа посвящена изучению действий редуктивных групп на нормальных аффинных алгебраических многообразиях с однопараметрическим семейством сферических орбит максимальной размерности при условии, что категорный фактор для такого действия одномерен. В качестве приложения полученных результатов мы завершаем классификацию действий группы $S L_{2}$ на нормальных трехмерных афффинных многообразиях. Основное поле $K$ предполагается алгебраически замкнутым и нулевой характеристики

Библиографофия: 15 названий.
\end{abstract}

\section{1. Введение}

Сложностью $c(X, G)$ действия $G: X$ линейной алгебраической группы $G$ на алгебраическом многообразии $X$ называют коразмерность орбиты общего положения для индуцированного действия борелевской подгруппы $B: X$ или, эквивалентно,

$$
c(X, G)=\operatorname{tr} \cdot \operatorname{deg} K(X)^{B} .
$$

Подробнее с понятием сложности можно познакомиться по работе [1].

Действия сложности нуль называют сферическими.

Для действий сложности один имеются две принципиально различные возможности:

1) на $X$ имеется открытая орбита группы $G$, имеюшая сложность 1 ;

2 ) группа $G$ действует на $X$ с однопараметрическим семейством сферических орбит максимальной размерности (будем в этом случае говорить, что $X$ является $q s$-многообразием (quasi-spherical variety) относительно действия групшы $G)$.

В этой работе мы будем рассматривать только вторую возможность.

Далее, если не оговорено противное, будем считать, что $G$ - связная редуктивная группа, регулярно действующая на неприводимом нормальном афффинном многообразии $X$.

Предположим, что орбита общего положения для такого действия сферична. Тогда и все орбиты группы $G$ на многообразии $X$ сферичны (см. [2]). Здесь мы покажем, что в этой ситуации при условии существования стабилизатора общего положения почти все замыкания типичной орбиты изоморфны, т.е. определено “замькание общего положения".

Работа выполнена при поддержке Международной соросовской программы образования в области точных наук, подпрограмма "Соросовские аспиранты” (грант № а96-423). 
Затем мы переходим к случаю действий сложности один со сферическими орбитами. Примерами таких действий служат вложения однородного пространства $K^{*} \times G / H$, где $H$ - алгебраическая сферическая подгруппа группы $G$ (однородное пространство $K^{*} \times G / H$ сферично по отношению к группе $K^{*} \times G$ и имеет сложность 1 при естественном действии группы $G$ ). Такие вложения могут быть классифицированы в рамках общей теории Луны-Вуста.

Будем назьвать нормальное аффинное $q s$-многообразиес условием $\operatorname{dim} X / / G=1$ $q s 1$-многообразием. Если действие $G: X$ стабильно, в частности, если $H$ содержит максимальный тор групшы $G$, условие $\operatorname{dim} X / / G=1$ выполняется автоматически. Мы покажем, что всякое бирационально тривиальное $q s 1$-многообразие $X$ можно получить с помощью определяемой ниже операции склейки из сферических вложений однородного пространства $K^{*} \times G / H$. Под бирациональной тривиальностью здесь подразумевается наличие в $X$ открытого $G$-инвариантного подмножества, изоморфного $G / H \times U$, где $U$-гладкая аффинная кривая с тождественньм действием группы $G$. Если группа эквивариантных автоморфизмов однородного пространства $G / H$, изоморфная $N_{G}(H) / H$, конечна, то склейка производится однозначно, и поэтому изучение такого класса действий сводится к хорошо разработанной теории сферических вложений. Для бирационально нетривиального действия доказывается, что оно может быть получено как фактор по действию конечной группы из бирационально тривиального действия с той же $G$-орбитой общего положения. Отсюда, в частности, вытекает рациональность особенностей $q s 1$-многообразий. Далее рассматривается класс $G$-многообразий, полученных с помощью операции склейки, но уже не обязательно над аффинной, а над любой гладкой алгебраической кривой. Оказьвается, этот класс $G$-многообразий характеризуется наличием "хорошего фактора" для действия группы $G$ в смысле работ Д. Мамфорда.

Затем изучаются свойства слоев морфизма факторизации для qs1-многообразий.

Полученные результаты позволят нам завершить классификацию действий группы $S L_{2}$ на нормальных неприводимых трехмерных аффинных алгебраических многообразиях. Напомним необходимые для этого сведения.

ЛЕмма 1.1 (см., например, [3, п. 4.1, лемма 2]). Любая алгебраическая подгруппа в $S L_{2}$ сопряжена одной из следующих подгрупп:

1) конечная подгруппа;

2) борелевская подгруппа $B$;

3) максимальный тор $T$;

4) нормализатор максимального тора $N$;

5) конечное расширение максимальной унипотентной подгруппы

$$
U_{n}=\left\{\left(\begin{array}{cc}
\varepsilon & a \\
0 & \varepsilon^{-1}
\end{array}\right) \mid a, \varepsilon \in K, \varepsilon^{n}=1\right\}, \quad n=1,2,3 \ldots
$$

ЛЕмма 1.2. Для всякого действия $S L_{2}$ существует стабилизатор общего положения (с.о.n.).

Пусть имеется действие $S L_{2}: X$ и многообразие $X$ трехмерно. Если с.о.п. такого действия есть конечная подгруппа, то $X$ содержит плотную орбиту группы $S L_{2}$. 
Такие действия классифицированы в работе [4], см. также [3]. Там показано, что локально транзитивное действие группы $S L_{2}$ на трехмерном нормальном аффинном многообразии есть либо транзитивное действие, либо определяется парой чисел из $\mathbb{N} \times\left(0, \frac{1}{2}\right]_{\mathbb{Q}}$. Первое из этих чисел определяет порядок стабилизатора общего положения, который в этой ситуации является циклической подгруппой. Второе число называется высотой и характеризует алгебру $U$-инвариантов.

Остаются еше три возможности:

1) с.о.п. $=U_{n}$ - назовем такие $S L_{2}$-многообразия $(S, U)$-многообразиями;

2) с.о.п. $=N$ - назовем такие $S L_{2}$-многообразия $(S, N)$-многообразиями;

3) с.о.п. $=T-$ назовем такие $S L_{2}$-многообразия $(S, T)$-многообразиями.

ЗАмечАниЕ. Случай с.о.п. $=B$ невозможен, так как $S L_{2} / B$ есть проективная прямая.

Всякое $(S, U)$-многообразие однозначно восстанавливается по спектру алгебры $U$-инвариантов, который представляет собой нормальную поверхность с естественным действием максимального тора группы $S L_{2}$. Более точно, справедлива

TEOPEMA 1 [2]. ( $S, U)$-многообразия взаимно однозначно соответствуют нормальныцм аффинныцм поверхностям $Y$ с фиксированной нетривиальной $\mathbb{Z}_{+}$-градуировкой на алгебре регулярных функций $K[Y]$. Эта градуировка задает действие одномерного тора $T$ на $Y$ и соответствующее $(S, U)$-многообразие изоморфно многообразию

$$
X=\left(Y \times K^{2}\right) / / T .
$$

При этом $K[X]^{S L_{2}} \cong K[Y]^{T}$.

Эта теорема сводит классификацию $(S, U)$-многообразий к классификации действий одномерного тора на нормальных поверхностях. По поводу последней см. [5].

Рассмотрим случай $(S, N)$-многообразий. Всякое такое многообразие бирационально тривиально. Вложение сферического однородного пространства $K^{*} \times S L_{2} / N$ задается парой взаимно простых натуральных чисел и склейка таких вложений производится над гладкой аффинной кривой однозначно. Поэтому для задания $(S, N)$-многообразия необходимо задать гладкую неприводимую афффинную кривую и в конечном множестве ее точек расставить отметки - пары взаимно простых натуральных чисел, см. теорему 3 ниже. В работе [2] этот результат был получен топологическими методами над полем комплексных чисел без использования теории Луны-Вуста.

Бирационально тривиальные $(S, T)$-многообразия описьваются абсолютно аналогично $(S, N)$-многообразиям. Остается разобрать случай бирационально нетривиальных $(S, T)$-многообразий. Каждое такое многообразие может быть получено как фактор по действию групшы $\mathbb{Z}_{2}$ из бирационально тривиального $(S, T)$-многообразия. Поэтому бирационально нетривиальное многообразие определяется гладкой неприводимой аффинной кривой с фиксированным нетождественным действием группы $\mathbb{Z}_{2}$ и $\mathbb{Z}_{2}$-инвариантной системой отметок, см. теорему 4.

Тем самым все нетождественные действия группы $S L_{2}$ на нормальных аффинных трехмерных алгебраических многообразиях описаны.

Гладкие $(S, N)$ - и $(S, T)$-многообразия описаны в работе [2]. 
Автору хотелось бы выразить искреннюю признательность своему научному руководителю профессору Э. Б. Винбергу за постановку задачи и постоянное внимание к работе. Много фактов, использованных при написании данной работы, я узнал в беседах с М. Брионом и $\Phi$. Кнопом во время конференции "Algebraic group actions", проходившей в Польской Республике в июне 1996 г.

В недавно написанной работе [6] разработан подход к классификации действий сложности один в терминах теории Луны-Вуста. Сопоставление этих результатов с результатами данной работы позволило исправить ряд неточностей в последней. Автор благодарит Д. А. Тимашева за критичное прочтение предварительной версии этого текста.

\section{2. Действия со сферической орбитой общего положения}

В следуюшем предложении условие нормальности $X$ несущественно.

ПрЕДЛОЖЕНИЕ 1 . Пусть $X$ есть $G$-многообразие, все $G$-орбитьи на X сферичны и существует стабилизатор общего положения с.о.п. $(G: X)=H$. Тогда почти все замыкания орбит типа $G / H$ (для открытого плотного множества орбит $G / H)$ изоморфнь некоторому вложению $Y$ сферического однородного пространства $G / H$.

ДокАЗАТЕЛЬСтво. Пусть $\Gamma(X)$ есть полугруппа старших весов $G$, соответствуюших неприводимым $G$-модулям, встречаюшимся в разложении алгебры $K[X]$ на неприводимые $G$-модули. Эта полугруппа конечно порождена по теореме Хаджиева (см. [7]). Пусть $\left\{\lambda_{1}, \ldots, \lambda_{k}\right\}$ - базис в $\Gamma(X)$. Зафиксируем некоторые старшие векторы $F_{\lambda_{1}}, \ldots, F_{\lambda_{k}} G$-модуля $K[X]$ весов $\lambda_{1}, \ldots, \lambda_{k}$, соответственно, и рассмотрим множество точек $X$, где ни одна из функций $F_{\lambda_{1}}, \ldots, F_{\lambda_{k}}$ не обрашается в нуль. Это множество открыто по Зарисскому. Отсюда следует, что замыкание орбиты общего положения есть аффинное многообразие с простьм спектром (так как $G / H$ сферично) и спектр этот есть $\Gamma(X)$. Остается показать, что умножение в алгебрах регулярных функций на таких замыканиях устроено одинаково. Это следует из того, что алгебра регулярных функций на замыкании орбиты типа $G / H$ есть $G$-инвариантная подалгебра в $K[G / H]$. Последняя алгебра имеет простой спектр и потому мультипликативная структура на подалгебре с заданным разложением на неприводимые $G$-модули определена однозначно. Предложение доказано.

ЗАмечАния. 1) Имеется гипотеза, согласно которой сферические подгрупшы в редуктивной группе не допускают непрерывных деформаций, и потому если есть действие со сферической орбитой обшего положения, то с.о.п. сушествует автоматически. Поэтому наше условие о сушествовании с.о.п. по-видимому не является ограничительным.

2) Даже при условии нормальности $X$ многообразие $Y$ может быть не нормальным. Например, рассмотрим действие одномерного тора на двумерной плоскости

$$
(x, y) \rightarrow\left(t^{2} x, t^{3} y\right)
$$

Здесь $Y$ - полукубическая парабола. Ниже мы покажем, что если $\operatorname{dim} X / / G=1$, то многообразие $Y$ нормально и совпадает со слоем общего положения для морфизма факторизации $\pi: X \rightarrow X / / G$. 


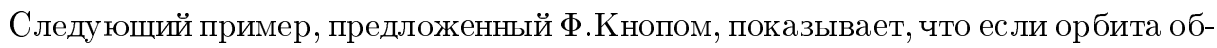
щего положения $G / H$ не сферична, то замыкания общего положения может не существовать.

Рассмотрим действие групшы $S L_{2}$ на трехмерном проективном пространстве $\mathbb{P}^{3}$ как на проективизации пространства матриц $M_{2 \times 2}$, на котором $S L_{2}$ действует левьги умножениями. В $\mathbb{P}^{3}$ есть открытая орбита типа $P S L_{2}$ и однопараметрическое семейство орбит $\mathbb{P}^{1} \times \mathbb{P}^{1}$, где группа $S L_{2}$ действует только на первом сомножителе.

Пусть $\Gamma$ - некоммутативная конечная подгруппа в $S L_{2}$. Профакторизуем пространство $\mathbb{P}^{3}$ по действию $\Gamma$, индуцированному действием группы $\Gamma$ на $M_{2 \times 2}$ умножениями справа. В $\mathbb{P}^{3} / \Gamma$ по-прежнему имеется однопараметрическое семейство $S L_{2}$-орбит $\mathbb{P}^{1} \times \mathbb{P}^{1}$. Рассмотрим произведение $\left(\mathbb{P}^{3} / \Gamma\right) \times \mathbb{P}^{1}$ с тривиальным действием $S L_{2}$ на втором сомножителе. Произведем раздутие подмногообразия $\mathbb{P}^{1} \times \Delta \subset \mathbb{P}^{1} \times \mathbb{P}^{1} \times \mathbb{P}^{1} \subset \mathbb{P}^{3} / \Gamma \times \mathbb{P}^{1}$, где $\Delta$ - диагональ в произведении второго и третьего сомножителей и группа $S L_{2}$ действует лишь на первом экземпляре $\mathbb{P}^{1}$.

На полученном многообразии вложения однородного пространства $S L_{2} / \Gamma$ соответствуют раздутиям различных орбит типа $S L_{2} / B$ и не могут быть почти все изоморфны в силу конечности группы эквивариантных автоморфизмов пространства $S L_{2} / \Gamma$.

Вложению полученного после раздутия проективного многообразия $P$ в проективное пространство соответствует некоторое очень обильное расслоение на $P$. Рассматривая достаточно высокую тензорную степень этого расслоения, можно добиться того, чтобы аффинный конус над $P$ был нормальньм аффинньм многообразием. Этот конус и является примером многообразия с орбитой общего положения типа $\left(K^{*} \times S L_{2}\right) / \Gamma$, где отсутствует замыкание общего положения.

Представляется интересным вопрос, для каких классов квазиаффинных однородных пространств замыкание общего положения существует.

\section{3. Основная конструкция}

Пусть $S$ - нормальное аффинное вложение сферического однородного пространства $K^{*} \times G / H$ групшы $K^{*} \times G$ такое, что $S / / G \cong A^{1}$. Возникает естественное действие $K^{*}$ на факторе $A^{1}$, единственную неподвижную точку которого будем считать нулем на прямой $A^{1}$. Прообраз в $S$ всякой ненулевой точки прямой $A^{1}$ есть сферическое $G$-многообразие $Y$. Назовем $Y$ типичнылм слоем для $S$.

Опишем некоторые операции, позволяющие получать новые $q s 1$-многообразия из уже имеющихся.

1. Операция перехода к расслоенному произведению.

Пусть $U$ есть гладкая аффинная кривая и задан этальный морфизм $\varphi: U \rightarrow A^{1}$ такой, что $\varphi^{-1}(0)=\{u\}$. Рассмотрим расслоенное произведение $X=S \times{ }_{A^{1}} U$

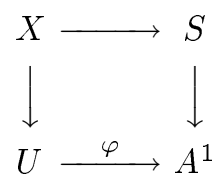

определенное условием $K[X]=K[U] \otimes_{K\left[A^{1}\right]} K[S]$. Многообразие $X$ нормально (следует из этальности $\varphi$ ) и является $q s 1$-многообразием с типичньм слоем $Y$. 


\section{2. Операция склейки.}

Пусть гладкая аффинная кривая $C$ покрыта конечным числом аффинных открытых по Зарисскому множеств $U_{j}$ так, что никакие два из них не совпадают, пересечение всех этих множеств есть открытое множество $U$ и $U_{j}=U \cup\left\{c_{j}\right\}, c_{j} \in C$ для любого $j$.

Пусть имеется набор афффинных многообразий $\left\{L_{j}\right\}$ и морфизмов $\left\{L_{j} \rightarrow U_{j}\right\}$ таких, что прообразы множества $U$ во всех многообразиях $L_{j}$ изоморфны друг другу, причем изоморфизмы согласованы с данными морфизмами. Тогда отождествим многообразия $L_{j}$ по этому изоморфному открытому множеству. Получим некоторое предмногообразие $L$ и согласно аффинному критерию из [8, с. 44] заданные морфизмы “склеиваются" в морфизм $L \rightarrow C$.

Изоморфизмы открытых кусков определены не канонически, поэтому результат склейки определен не однозначно. Будем говорить, что $L$ получено из многообразий $L_{j}$ операцией склейки, если $L$ получено с помощью описанной выше конструкции при некотором выборе изоморфизмов изоморфных открытых подмножеств.

В нашей ситуации пусть $\left\{S_{j}\right\}$ есть набор сферических вложений для $K^{*} \times G / H$ с одним и тем же типичным слоем $Y$. Если для всякого $U_{j}$ задан этальный морфизм, использованный в операции перехода к расслоенному произведению, над каждым $U_{j}$ можно построить соответствуюшее расслоенное произведение $X_{j}$ и затем отождествить ("склеить") их по изоморфному куску $U \times Y$. Получим некоторое предмногообразие $X$.

ЛЕмма 3.1. Предмногообразие $X$ отделимо, т.е. является алгебраическим многообразием.

ДоКАЗАТЕЛЬСТво. Известно (см. [8, с. 50], что отделимость предмногообразия $X$ эквивалентна следуюшему свойству: для любых двух морфизмов $\varphi, \psi: Y \rightarrow X$ ( $Y$ - любое предмногообразие) множество $\{y \in Y \mid \varphi(y)=\psi(y)\}$ замкнуто в $Y$. Там же показано, что если любые две точки из $X$ лежат в одном открытом аффинном подмножестве, то $X$ отделимо. Пусть для некоторых морфизмов $\varphi, \psi: Y \rightarrow X$ множество $Z=\{y \in Y \mid \varphi(y)=\psi(y)\}$ и $z \in \bar{Z} \backslash Z$. Точки $\varphi(z)$ и $\psi(z)$ не могут лежать в одном из склеиваемых аффинных многообразий, поскольку это противоречит отделимости этого аффинного многообразия. Поэтому можно считать, что $\varphi(z) \in \pi^{-1}\left(c_{j_{1}}\right)$ и $\psi(z) \in \pi^{-1}\left(c_{j_{2}}\right), j_{1} \neq j_{2}$. Рассмотрим композиции морфизмов $\pi \circ \varphi, \pi \circ \psi: Y \rightarrow C$. Поскольку $C$ есть отделимое многообразие, множество $Z^{\prime}=\{y \in Y \mid(\pi \circ \varphi)(y)=(\pi \circ \psi)(y)\}$ замкнуто в $Y$ и $z \notin Z^{\prime}$. С другой стороны ясно, что $Z \subset Z^{\prime}$. Полученное противоречие доказывает лемму 3.1 .

Аффинность многообразия $X$ вытекает из следуюшей леммы.

ЛЕмМа ОБ АФФИНном МОРФИЗМЕ (см. [9]). Пусть $f: X \rightarrow Y-$ морфизм алгебраических многообразий такой, что существует открытое покрытие $\left\{V_{i}\right\}$ многообразия $Y$ u $f^{-1}\left(V_{i}\right)$ аффинно для кажсдого $i$. Тогда для любого аффинного открытого множества $V \hookrightarrow Y f^{-1}(V)$ - аффинно.

ПРЕДЛОЖЕНИЕ 2. Если группа $\operatorname{Aut}_{G} Y$ конечна, то склейка определена однозначно.

Для доказательства нам потребуется 
ЛЕмма 3.2. Всякий G-автоморфизм многообразия $Y$ однозначно продолжается до $G$-автоморфизма многообразия $S$, тождественного на фактоpe $S / / G$.

ДокАЗАТЕЛЬСТво. Поскольку $K[Y]$ есть $G$-модуль с простым спектром, всякий $G$-автоморфизм действует на каждом его неприводимом подмодуле умножением на некоторую константу. Этот автоморфизм естественным образом продолжается на $K\left[\left(A^{1} \backslash\{0\}\right) \times Y\right]$, а затем и на $K[S]$ как на $G$-инвариантную подалгебру. Лемма 3.2 доказана.

В силу конечности группы $\operatorname{Aut}_{G} Y$ отождествление по изоморфному открытому подмножеству многообразий $S_{j_{1}}$ и $S_{j_{2}}$ задается одним элементом группы $\operatorname{Aut}_{G} Y$ и потому единственно с точностью до изоморфизмов склеиваемых многообразий. Предложение 2 доказано.

ЗАмечание. Если группа $\operatorname{Aut}_{G} Y$ бесконечна, склейка может быть осуществлена многими способами. Так если $G$ - одномерный тор, вопрос о числе способов отождествления есть вопрос о числе локально тривиальных в топологии Зарисского линейных расслоений над гладкой аффинной кривой.

Покажем, что предложенная конструкция склейки является в некотором смысле универсальной.

ОПРЕДЕЛЕНИЕ. Назовем $q s 1$-многообразие $X$ nростым, если выполнены следующие условия:

(1) сушествует с.о.п. $(G: X)=H$;

(2) многообразие $X$ имеет тривиальный бирациональный тип, т.е. содержит открытое $G$-инвариантное подмножество $X_{0}$, изоморфное $G / H \times U$ для некоторой гладкой кривой $U$.

Условие (2) эквивалентно тривиальности некоторого класса когомологий Галуа. Если $G$ есть тор, то это условие выполнено для любого действия. Можно показать, что условие (2) выполнено, если группа $N_{G}(H) / H$ связна, см. $[7$, п. 2.7].

Следуюшее предложение показывает, что каждое $q s 1$-многообразие можно получить из простого qs1-многообразия факторизацией по конечной группе.

ПРЕДЛОЖЕНИЕ 3. Для любого qs1-многообразия $X$ со стабилизатором общего положения $H$ найдется простое qs1-многообразие $\widetilde{X}$ с тем же стабилизатором общего положения и конечная группа $F$, действующая на $\widetilde{X}$ регулярными $G$-эквивариантными автоморфизмами так, что

$$
X \cong \widetilde{X} / / F \text {. }
$$

ДокаЗАТЕЛЬСтво. Пусть $X_{1} \subset X$ - открытое подмножество, состоящее из точек, стабилизаторы которых сопряжены $H$, и $X_{1}^{H} \subset X_{1}$ - множество $H$-неподвижных точек. Рассмотрим произвольную кривую в $X_{1}^{H}$, пересекаюшую $N_{G}(H)$-орбиты общего положения в $X_{1}^{H}$ трансверсально. Эта кривая определяет квазисечение для действия $G: X$. Имеется естественный доминантный морфизм $G / H \times U \rightarrow X$. Рассмотрим расширение $K(X / / G) \subset K(U)$ и продолжим его до расширения Галуа $K(X / / G) \subset K(U) \subset K(\widetilde{U})$ с группой Галуа $F$. 
Пусть $\widetilde{K[X]}$ есть целое замькание $K[X]$ в $K(G / H \times \widetilde{U})$ и $\widetilde{X}=\operatorname{Spec} \widetilde{K[X]}$. Из конечности морфизма $\widetilde{X} \rightarrow X$ вытекает его сюръективность. Учитывая нормальность $X$, мы получаем $X \cong \widetilde{X} / / F$. Наконец тот факт, что $\widetilde{X}$ бирационально изоморфно $G / H \times \widetilde{U}$, вытекает из следующей стандартной леммы.

ЛЕмма 3.3. Пусть $А$ - конечно порожденная челостная алгебра над полем $K$ и $F_{1}=Q A$ - ее поле частных. Если $F_{1} \subset F_{2}$ - конечное расширение полей и $B$ - целое замыкание $A$ в поле $F_{2}$, mо $Q B=F_{2}$.

Вернемся к простым qs1-многообразиям. Обозначим через $Y$ замыкание орбиты $G$ / $H$ обшего положения, которое существует согласно предложению 1 . Алгебра регулярных функций на $X_{0}$ есть $K[U] \bigotimes_{K} K[G / H]$ и алгебра $K[G / H]$ имеет простой спектр. Уменышив если нужно $U$, можно считать, что в $X$ есть открытое $G$-инвариантное подмножество, изоморфное $U \times Y$. Далее через $X_{0}$ будем обозначать это подмножество.

Обозначим через $C=X / / G$ гладкую аффинную кривую $\operatorname{Spec} K[X]^{G}$ (гладкость следует из нормальности $X)$. Для морфизма факторизации $\pi: X \rightarrow C$ все компоненты слоев имеют коразмерность 1 в $X$. Следуюшее предложение показывает, что кривую $U$ можно рассматривать как подмножество в $C$ и $\pi^{-1}(U)=X_{0} \cong$ $U \times Y$.

ПРЕДЛОЖЕНИЕ 4. Пусть $f: X \rightarrow X_{1}$ есть доминантный морфизм неприводимых нормальных аффинных многообразий и слои общего положсения этого морфизма связны (для морфизма факторизации по связной редуктивной группе последнее условие выполнено). Тогда слои общего положсения неприводимыл.

ДокАЗАтЕльство. Пусть $\widehat{K\left(X_{1}\right)}$ - алгебраическое замькание поля $K\left(X_{1}\right)$ в $K(X)$. Если $\widehat{K\left(X_{1}\right)} \neq K\left(X_{1}\right)$, то по лемме 3.3 и $\widehat{K\left[X_{1}\right]} \neq K\left[X_{1}\right]$, где $\left.\widehat{K\left[X_{1}\right.}\right]$ - целое замыкание $K\left[X_{1}\right]$ в $K(X)$. Из нормальности $X$ вытекает включение $\widehat{K\left[X_{1}\right]} \subset K[X]$ и потому имеются регулярные морфизмы $X \rightarrow \widetilde{X}_{1} \rightarrow X_{1}$, где последний морфизм конечен и небирационален. Это противоречит условию связности слоев.

В случае $\widehat{K}\left(X_{1}\right)=K\left(X_{1}\right)$ см. [10, с. 172]. Предложение доказано.

ЗАмЕчАниЕ. Без условия нормальности $X$ утверждение предложения неверно - можно рассмотреть поверхность $x^{2}=y^{2} z$ и проекцию на прямую с координатой $z$.

Пусть $K[Y]=\bigoplus_{\lambda} V_{\lambda}$ - изотипное разложение. Тогда $K\left[X_{0}\right]=\bigoplus\left(K[U] \otimes_{K} V_{\lambda}\right)$ и потому в силу $G$-инвариантности $K[X]=\bigoplus\left(T_{\lambda} \otimes_{K} V_{\lambda}\right)$, где $T_{\lambda} \subset K[U]$.

Алгебра $K[X]$ конечно порождена и можно считать ее порожденной элементами вида $c_{\lambda_{i}} v_{\lambda_{i}}, i=1, \ldots, s, v_{\lambda_{i}} \in V_{\lambda_{i}}, c_{\lambda} \in K[U]$. Уменьшая множество $U$, мы можем считать, что $c_{\lambda_{i}} \in K[U]^{*}$.

Пусть $C \backslash U=\left\{y_{1}, \ldots, y_{t}\right\}$. Можно считать, вновь (если нужно) уменьшая $U$, что:

1) $U_{j}=U \bigcup\left\{y_{j}\right\}$ аффинно;

2) существует $\pi_{j} \in R_{j}$ такое, что $\pi_{j}=\varrho_{j}^{*}, \varrho_{j}: U_{j} \rightarrow A^{1}-$ этально и $\varrho_{j}^{-1}(0)=\left\{y_{j}\right\}$ (здесь $\left.R_{j}=K\left[U_{j}\right]\right)$. 
Обозначим через $R$ кольцо $K[U]$. Тогда $\pi_{j} R_{j}=$ идеал $y_{j}$ и $R^{*}=R_{j}^{*} \times\left\{\pi_{j}^{k}\right\}_{k \in \mathbb{Z}}$. Пусть $X_{j}=\pi^{-1}\left(U_{j}\right)$. Тогда

$$
K\left[X_{j}\right]=R_{j}\left[\ldots, \pi_{j}^{r_{l}} V_{\lambda_{l}}, \ldots\right]
$$

для некоторого конечного набора пар $P_{j}=\left\{\left(r_{l}, \lambda_{l}\right) \in \mathbb{Z} \times \Gamma(X)\right\}$.

Рассмотрим многообразие

$$
S_{j}=\operatorname{Spec} K\left[t, \ldots, t^{r_{l}} V_{\lambda_{l}}, \ldots\right], \quad K\left[t, \ldots, t^{r_{l}} V_{\lambda_{l}}, \ldots\right] \subset K[t] \otimes K[Y] .
$$

На $S_{j}$ определено естественное действие одномерного тора (на переменной $t$ ) и потому $S_{j}$ становится сферическим многообразием для группы $K^{*} \times G$.

Имеет место коммутативная диаграмма:

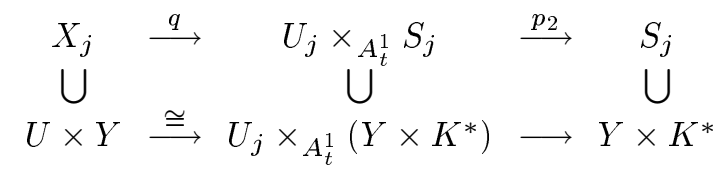

и отображение $q$ есть изоморфизм. Поскольку $\varrho_{j}: U_{j} \rightarrow A^{1}$ этально, проекция $p_{2}$ также этальна. В частности, $S_{j}$ нормально.

Итак, многообразие $X$ получается переходом к расслоенным произведениям от сферических многообразий $S_{j}$ к $X_{j}$ и последуюшей склейкой $X_{j}$. В наших рассуждениях мы следовали работе [5], где подобные рассмотрения проводятся в торическом случае. Нами доказана:

Tеорема 2.1. Всякое простое qs1-многообразие со стабилизатором общего положения $H$ может быть получено операчией склейки из нормальных сферических вложений $S_{j}$ однородного пространства $K^{*} \times G / H$ с одним и тем же типичным слоем $Y$ и условием $S_{j} / / G=A^{1}$.

ЗАмЕчАниЕ. В духе работы [5] можно сказать, что простые $q s 1$-многообразия сфероидальньь.

СлеДСтвИЕ. Особенности qs1-многообразия рациональныь.

Действительно, для сферических многообразий рациональность особенностей показана в [11] и следствие вытекает из этальности построенных морфизмов и предложения 4 , так как рациональность особенностей сохраняется при факторизации по конечной (и вообше редуктивной) группе, см. [7, п. 3.9].

Далее предполагается, что читатель знаком с понятием цветного конуса. С этим понятием можно познакомиться по работе [12].

Пусть задан конечный набор цветных конусов $\mathrm{C}_{j}$, определяюших вложения $S_{j}$ однородного пространства $K^{*} \times G / H$. Склейка этих вложений над некоторой кривой в одно простое $q s 1$-многообразие возможна тогда и только тогда, когда выполнены следующие условия: (а) $S_{j} / / G=A^{1}$; (б) типичный слой у всех вложений $S_{j}$ изоморфен некоторому $G / H$-вложению $Y$ для всех $j$; (в) $S_{j}$ афффинны. 
ОПРЕДЕЛЕНИЕ. Назовем $q s$-многообразие $X$ жестким, если для стабилизатора общего положения $H$ выполнено условие $N_{G}(H)=H$.

Если сферическое однородное пространство $G / H$ квазиаффинно и группа $N_{G}(H) / H$ конечна, то из результатов М. Бриона и $\Phi$. Кнопа вытекает, что подгруппа $H$ редуктивна, см., например, [13, cor. 7.6]. Согласно критерию Луны замкнутости орбит однородное пространство $G / H$ в этом случае допускает лишь одно аффинное вложение, состояшее из единственной орбиты $G / H$. Поэтому $G$-орбиты общего положения на $X$ замкнуты и всякое жесткое $q s$-многообразие является qs1-многообразием. Всякое жесткое $q s$-многообразие бирационально тривиально.

Для жестких $q s$-многообразий теорема 2.1 с учетом предложения 2 может быть переформулирована следующим образом:

Теорема 2.2. Всякое жесткое qs-многообразие $X$ со стабилизатором общего положсения $Н$ однозначно определяется следующим набором данных:

1) гладкая аффинная кривая $C$ (которая есть фактор $X / / G)$;

2) конечный набор $P$ попарно различных точек кривой $C$ (возмохсно, пустой);

3) каждой точке множсетва $P$ поставлен в соответствие иветной конус для однородного пространства $K^{*} \times G / H$, не лежащий в подпространстве, соответствующем $G / H$ и задающий аффинное вложение пространства $K^{*} \times G / H$.

Обратно, всякий такой набор данньх определяет жесткое qs-многообразие группь $G$.

ЗАмЕчАниЕ. В работе [12] указан критерий, позволяющий легко выяснять, афффинно ли вложение, отвечающее данному цветному конусу.

СлЕДСТВИЕ. Если в условиях теоремы 2.2 дополнительно известно, что ранг однородного пространства $G / H$ равен единице, то с кажсдй точкой множества $P$ вместо цветного конуса достаточно связывать пару взаимно простых натуральных чисел. В этом случае все слои морфизма факторизачии $\pi: X \rightarrow C$ неприводимы.

ДоказАТЕЛьство. Известно (см. [12]), что размерность конуса инвариантных нормирований $\Upsilon_{G / H}$ равна рангу соответствуюшего однородного пространства и при условии конечности группы $N_{G}(H) / H$ конус $\Upsilon_{G / H}$ строго выпуклый. Можно отождествить $\Upsilon_{G / H}$ с отрицательньп лучом координатной прямой и потому конус $\Upsilon_{K^{*} \times G / H}$ совпадет с левьп полупространством (рис. 1 ).

Множество красок у пространства $G / H$ непусто, иначе $G / H$ допускает как минимум два аффинных вложения $(0, \varnothing)$ и $\left(\Upsilon_{G / H}, \varnothing\right)$. Не все краски расположены слева, иначе имелся бы аффинный конус $\left(\Upsilon_{G / H}, \rho(D)\right)$. Однородное пространство $G / H$ аффинно, поэтому все его краски лежат справа. Применяя автоморфизм тора $K^{*}: t \rightarrow t^{-1}$, можно считать, что наш цветной конус расположен в верхней полуплоскости. Он должен содержать все краски пространства $G / H$ и потому для его задания необходимо и достаточно задать один луч в левом верхнем квадранте. Поскольку объемлющее пространство задано над полем рациональных чисел, такой 


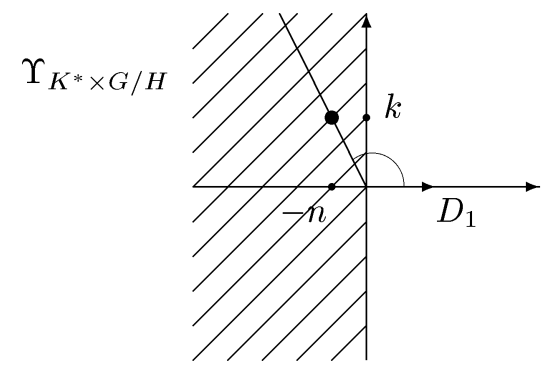

Рис. 1

луч однозначно определяется парой взаимно простых натуральных чисел. Заданньй луч отвечает неприводимому дивизору, который есть слой морфизма факторизации над особой точкой, и поэтому все слои неприводимы. Следствие доказано.

Ясно, что склейка аффинных вложений $S_{j}$ возможна не только над аффинной, но и над произвольной гладкой алгебраической кривой. Выясним, какой класс $G$-многообразий сложности один так получается.

В геометрической теории инвариантов Д. Мамфорда было введено понятие “хорошего фактора" (в англоязычной литературе - "good quotient").

ОПрЕДЕЛЕНИЕ. Пусть $X$ - алгебраическое $G$-многообразие. Морфизм $\pi: X \rightarrow C$, где $C$ - алгебраическое пространство, назьвается "хорошим фактором", если

а) морфизм $\pi G$-эквивариантен относительно данного действия $G$ на $X$ и тождественного действия $G$ на $C$;

б) $\pi$ есть афффинньй морфиизм;

в) $\pi_{*}\left(O_{X}^{G}\right) \cong O_{C}$.

Условие б) означает, что на $C$ найдется конечное аффинное покрытие такое, что его прообраз есть аффинное покрытие $X$. "Хороший фактор" является категорным фактором и потому определен однозначно с точностью до канонического изоморфизма. Существование такого фактора для произвольных действий на алгебраических многообразиях - явление редкое. Для этого необходимо, в частности, чтобы у каждой точки из $X$ сушествовала открытая аффинная инвариантная окрестность. В случае, когда $X$ аффинно, “хороший фактор" $\pi: X \rightarrow \operatorname{Spec}\left(K[X]^{G}\right)$ существует всегда.

Известно, что “хороший фактор” получается из фактора Мамфорда для множества полустабильных точек при некотором вложении $X$ в проективное пространство тогда и только тогда, когда $C$ есть квазипроективное многообразие. В более общей ситуации "хороший фактор" изучался в работах А. Бяльницкого-Бирули и Й. Свецицкой, см. [14] и библиографию там же. В этих работах рассматривалась проблема описания всех открытых инвариантных подмножеств алгебраического $G$-многообразия, обладающих "хорошим фактором".

Мы можем достаточно конструктивно описать некоторый класс $G$-многообразий, обладаюших “хорошим фактором”, безотносительно к какому-либо вложению. 
ТЕОРЕМа 2.3. Нормальное алгебраическое бирационально тривиальное $G$-многообразие $X$ сложсности один со стабилизатором общего полохсения H допускает "хороиий фактор" на гладкую алгебраическую кривую $C$ тогда и только тогда, когда оно может бъть получено склейкой над кривой $C$ нормальных аффинных сферических вложений $S_{j}$ однородного пространства $K^{*} \times G / H$ с одним и тем же типичным слоем $Y$ и условием $S_{j} / / G=A^{1}$.

Для доказательства теоремы 2.3 достаточно выбрать покрытие кривой $C$, как при проведении основной конструкции, и воспользоваться леммой об аффинном морфизме.

Поскольку всякая алгебраическая кривая квазипроективна, данный фактор можно получить с помощью конструкции Мамфорда при подходящем вложении в проективное пространство.

СлЕДСТВИЕ. Всякое многообразие из теоремы 2.3 квазипроективно.

\section{4. Свойства слоев морфизма факторизации}

Теорема 2.1 сводит изучение многих геометрических вопросов о многообразиях сложности 1 с однопараметрическим семейством сферических орбит к вопросам о сферических многообразиях, которые хорошо изучены. Рассмотрим вопрос о нормальности и неприводимости слоев морфизма факторизации для простых qs1-многообразий.

Обозначим через $\omega$ операцию перехода от $G$-алгебры к подалгебре еe $U$-инвариантов, где $U$ есть максимальная унипотентная подгруппа в $G$, т.е. $\omega: K[X] \rightarrow$ $K[X]^{U}$. Той же буквой будем обозначать аналогичный переход на уровне $G$-многообразий: $\omega: X \rightarrow \operatorname{Spec} K[X]^{U}$. Если $X$ есть qs1-многообразие для групшы $G$, Spec $K[X]^{U}$ есть $q s 1$-многообразие для максимального тора $T$ группы $G$, причем операция $\omega$ коммутирует с переходом к алгебре регулярных функций на некотором слое морфизма факторизации. Учитывая что целозамкнутость и целостность алгебры есть устойчивые свойства в терминах работы [11], неприводимость и нормальность слоев для $X$ эквивалентны неприводимости и нормальности слоев для $T$-многообразия $\operatorname{Spec} K[X]^{U}$. Поэтому можно ограничиться действиями торов и в силу теоремы 2.1 достаточно изучить случай, когда $X$ есть торическое многообразие для $(n+1)$-мерного тора, где $n=\mathrm{rk} G$.

Торическое многообразие однозначно задается выпуклым конусом $C$ в $\mathbb{Q}^{n+1}$, линейная оболочка которого совпадает со всем $\mathbb{Q}^{n+1}$ и которьй порожден векторами, координаты которых соответствуют всевозможным весам тора $\left(K^{*}\right)^{n+1}$, встречающимся в весовом разложении алгебры $K[X]$. Такой конус является двойственным по отношению к конусу, который обычно рассматривается в теории торических многообразий. Условие $X / / T=A^{1}$ соответствует тому, что $C$ содержит положительный луч последней координатной оси в $\mathbb{Q}^{n+1}$ и не содержит отрицательного луча (мы предполагаем, что $T \subset\left(K^{*}\right)^{n+1}$ по первым $n$ координатам).

ОПРЕДЕЛЕНИЕ. Будем говорить, что действие $T: X$ есть $f p$-действие (fixed-point action), если проекция $C_{0}$ конуса $C$ на $\mathbb{Q}^{n}$ (первые $n$ координат) лежит в некотором замкнутом полупространстве. В противном случае будем говорить о $n f p$-действии. 
Если группа $G$ полупроста, то индуцированное действие $T: \operatorname{Spec}\left(K[X]^{U}\right)$ является $f p$-действием.

Лемма 4.1. Все слои морфизма факторизачии $\pi: X \rightarrow X / / T=A^{1}$, кроме слоя над нулем, являются торическими многообразиями относительно тора $T$, соответствующими конусу $C_{0}$.

$$
\begin{gathered}
\text { ДоказАтЕЛЬСтво. Имеем } K[X]=K\left[t, \ldots, t^{r_{l}} \chi^{\lambda_{l}}, \ldots\right] . \text { Тогда } \\
K\left[\pi^{-1}\left(A^{1} \backslash\{0\}\right)\right]=K[X]\left[t^{-1}\right]=K\left[t, t^{-1}\right] \otimes_{K} K\left[\ldots, \chi^{\lambda_{l}}, \ldots\right]=K\left[t, t^{-1}\right] \bigotimes_{K} K[Y] .
\end{gathered}
$$

Из леммы 4.1 следует, что $n f p$-действия характеризуются тем, что слой общего положения для морфизма $\pi: X \rightarrow X / / T$ есть сам тор $T$.

ЗАмЕчАниЕ. Если у нас имеется конечньй набор конусов в $\mathbb{Q}^{n+1}$, содержащих только положительный луч последней координатной оси и проекции которых на $\mathbb{Q}^{n}$ совпадают, то соответствующие этим конусам торические многообразия можно склеить в одно многообразие $X$ (неоднозначно!) и всякое нормальное аффинное $(n+1)$-мерное многообразие с эффективным действием $n$-мерного тора и с одномерным фактором может быть получено так.

Остается рассмотреть слой $Y_{0}$ морфизма $\pi$ над нулем. На алгебре $K[Y]$ имеется корректно определенная $\mathbb{Z}$-фильтрация: сопоставим каждому весу $\left(q_{1}, \ldots, q_{n}\right)$, $q_{i} \in \mathbb{Z}$, из $C_{0}$ минимальное из чисел $q_{n+1} \in \mathbb{Z}$ такое, что $\left(q_{1}, \ldots, q_{n}, q_{n+1}\right) \in C$.

Переход от алгебры $K[X]$ к алгебре $K\left[Y_{0}\right]$ заключается в факторизации по идеалу $(t)$, что можно интерпретировать как переход от $K[Y]$ к присоединенной алгебре gr $K[Y]$ относительно введенной вьше фильтрации с последуюшей факторизацией по максимальному нильпотентному идеалу. После этого в $K[Y]$ останутся лишь те веса, которые являются проекциями целочисленных точек, лежащих на собственных гранях конуса $C$. Произведение собственных относительно тора функций, отвечающих точкам, лежащим на различных гранях конуса $C$, будет отвечать точке, лежашей внутри конуса $C$, и потому после сужения на нулевой слой станет нильпотентной функцией.

Отсюда легко видеть, что число неприводимых компонент слоя $Y_{0}$ равно числу проекций максимальной размерности граней конуса $C$ на $\mathbb{Q}^{n}$. Они определяют разбиение конуса $C_{0}$ на конусы той же размерности.

ПРЕДЛОЖЕНИЕ 5. 1) Для $n f p$-действий число неприводимых компонент слоя над нулевой точкой равно числу граней конуса $C$.

2) Для $f p$-действий число неприводимых компонент слоя над нулевой точкой меньше или равно числу граней конуса $C$ минус $n$.

СлЕДСТвИЕ 1. При действии полупростой группы ранга 1 или при $f p$-действии одномерного тора все слои морфизма факторизаџии неприводимыb.

Тем самым получено еше одно доказательство предложения 5 работы [2].

СлЕДСТВИЕ 2. Для $n f p$ действия $n$-мерного тора на $(n+1)$-мерном нормальном аффинном многообразии следующие условия эквивалентны:

1) все слои морфизма факторизации неприводимь;

2) все слои морфизма факторизации изоморфны самому п-мерному тору. 
В случае $f p$-действий неприводимость всех слоев означает, что соответствующий конус симплициален.

Заметим также, что при действии $n$-мерного тора на $(n+1)$-мерном многообразии доопределить это действие до локально-транзитивного действия $(n+1)$-мерного тора можно, как правило, неединственным способом, однако число граней получающегося конуса постоянно.

Рассмотрим некоторую проекцию максимальной размерности $C_{0, i}$ некоторой грани $C_{i}$ конуса $C, C_{0, i} \subset C_{0}$. Пусть грань $C_{i}$ выделена на конусе $C$ условием $a_{1} x_{1}+\cdots+a_{n} x_{n}+a_{n+1} x_{n+1}=0$. Тогда проекции целочисленных точек грани $C_{i}$ выделяются среди всех точек с целыми координатами, попавшими в $C_{0, i}$, условием $\frac{a_{1} x_{1}+\cdots+a_{n} x_{n}}{a_{n+1}} \in \mathbb{Z}$, т.е. принадлежат подрешетке конечного индекса. Потому неприводимая компонента нулевого слоя, соответствуюшая грани $C_{0, i}$, есть торическое многообразие, но уже не для самого тора $T$, а для его фактора по конечной подгруппе, определенной указанной подрешеткой. Отсюда следует

ПРЕДЛОЖЕНИЕ 6. Пусть имеется простое qs1-действие редуктивной групnъи G. Тогда все неприводимые компоненты каждого из слоев морфизма факторизации нормальны.

Перейдем теперь к случаю $G=S L_{2}$.

\section{5. Классификация $(S, N)$-многообразий}

Рассмотрим классификацию аффинных нормальных вложений однородного пространства $K^{*} \times S L_{2} / N$ в рамках теории Луны-Вуста.

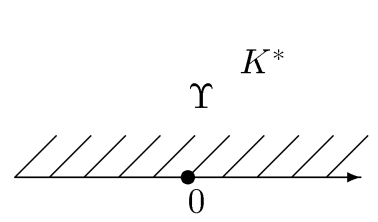

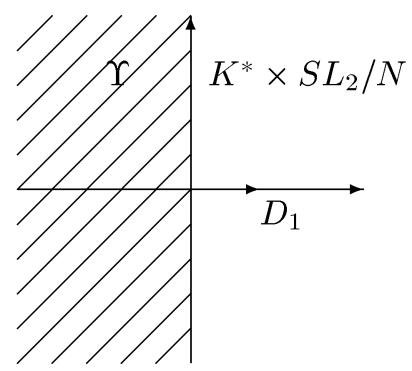

Рис. 2

На рис. 2 изображены конус инвариантных нормирований $\Upsilon$ и “краски” для однородных пространств $K^{*}, S L_{2} / N, K^{*} \times S L_{2} / N$, соответственно. Необходимые определения см. в [12]. Критерий аффинности из [12] показывает, что все нормальные афффинные вложения однородного пространства $K^{*} \times S L_{2} / N$ задаются цветными конусами одного из типов, изображенных на рис. 3.

Первые два вложения есть $S L_{2} / N \times K^{*}$ и $S L_{2} / N \times K$, соответственно. Для задания конуса типа 3 ) достаточно задать прямую в верхнем левом квадранте, определенную над полем рациональных чисел. Задание такой прямой эквивалентно заданию пары взаимно простых натуральных чисел $(n, k)$.

Вложения, соответствуюшие конусам типа $2^{\prime}$ ) и $3^{\prime}$ ), получаются из вложений для конусов 2) и 3 ) действием автоморфизма $t \rightarrow t^{-1}$ на торе $K^{*}$. 
1)

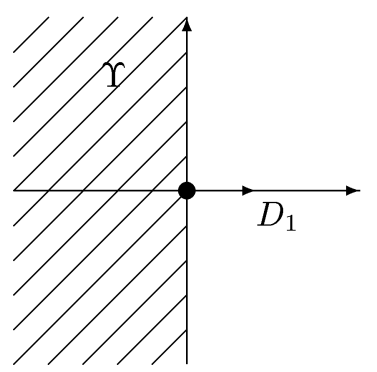

$(0, \varnothing)$
2)

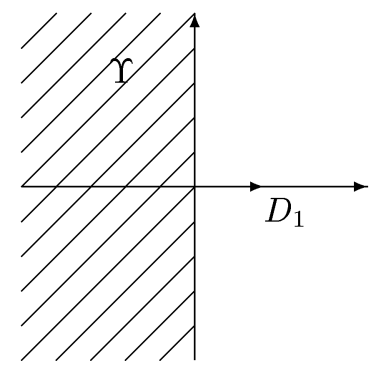

$\left(\mathbb{Q}^{+}(0,1), \varnothing\right)$ $\left.2^{\prime}\right)$

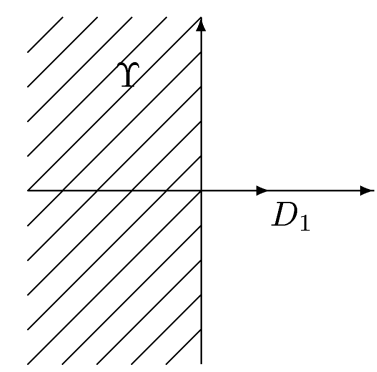

$\left(\mathbb{Q}^{+}(0,-1), \varnothing\right)$
3)

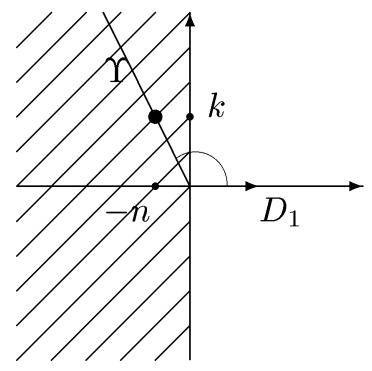

$\left.3^{\prime}\right)$

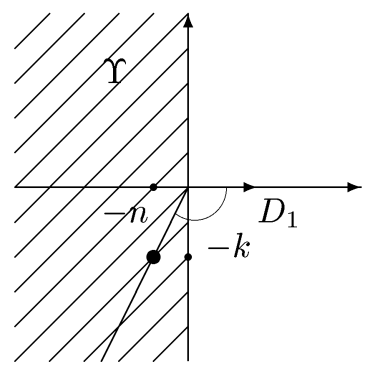

Рис. 3

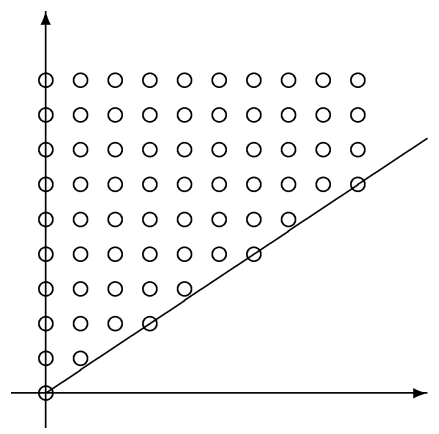

Рис. 4

Явно алгебру регулярных функций на многообразии, отвечающем вложению, заданному парой $(n, k)$, можно описать так, как показано на рис. 4.

Рассмотрим конус $C$ в $\mathbb{Q}^{2}$, заключенный между положительным лучем оси $O y$ и лучем, исходящим из начала координат и проходящим через точку с координатами $(k, n)$ (напомним, что мы предполагаем $k>0, n>0$ ). Тогда

$$
K[X]=\left\{t^{r_{2}} V_{4 r_{1}} \mid\left(r_{1}, r_{2}\right) \in C, r_{1}, r_{2} \in \mathbb{Z}\right\} \subset K\left[A^{1}\right] \otimes K\left[S L_{2} / N\right],
$$

где через $V_{4 r_{1}}$ обозначен $\left(4 r_{1}+1\right)$-мерный неприводимый $S L_{2}$-модуль. Нормальность полученного многообразия $X$ вытекает из того, что по построению $\operatorname{Spec} k[X]^{U}$ есть торическое многообразие. 
Воспользовавшись склейкой и предложением 2 , получаем следуюшую теорему.

ТЕОРЕМА 3. $(S, N)$-многообразия находятся во взаимно однозначном соответствии со следующим набором данных:

1) $C$ - гладкая неприводимая аффинная кривая;

2) $W$ - конечныци набор из $m$ точек на этой кривой (возможнно, $m=0$ );

3) набор пар взаимно простых натуральных чисел $\left(k_{1}, n_{1}\right), \ldots,\left(k_{m}, n_{m}\right)$, поставленных по одной паре у каждой точки из множества $W$ иназываемых отметками этих точек.

Утверждение теоремы 3 является частным случаем для следствия из теоремы 2.2, однако здесь можно предъявить геометрическую реализацию многообразия, отвечающего паре чисел $(n, k)$. Соответствующее многообразие построено в работе [2], где оно обозначено Norm $X_{n}^{k}$. Напомним необходимые сведения из работы [2]. Пусть $V_{2}$ - модуль присоединенного представления для групшы $S L_{2}$, a $X^{k}$ есть фактор пространства $V_{2}$ по группе $\mathbb{Z}_{2 k}$, действующей скалярно умножениями на корни степени $2 k$ из единицы. Если обозначить стандартные координаты на $V_{2}$ как $a, b, c$, то $K\left[X^{k}\right]=K\left[a^{i} b^{j} c^{l} \mid i+j+l=2 k\right]$. Рассмотрим многообразие $X_{n}^{k}$, являюшееся спектром алгебры $K\left[a^{i} b^{j} c^{l}(i+j+l=2 k), z \mid z^{n}=\left(b^{2}-4 a c\right)^{k}\right]$. Действие группы $S L_{2}$ естественным образом индуцируется с модуля $V_{2}$ на все построенные многообразия, элемент $z$ является инвариантным. Морфизм нормализации Norm $X_{n}^{k} \rightarrow X_{n}^{k}$ биективен. Выясним, какой конус соответствует вложению $\operatorname{Norm} X_{n}^{k}$

Обозначим через $\epsilon$ фундаментальньй вес тора $K^{*}$, а через $\lambda$ фундаментальньй вес максимального тора групшы $S L_{2}$. Поскольку $B$-инвариантный дивизор однородного пространства $K^{*} \times S L_{2} / N$ содержит в своем замыкании единственную $S L_{2}$-неподвижную точку многообразия Norm $X_{n}^{k}$, “краска" $D_{1}$ в данньй конус входит. На многообразии Norm $X_{n}^{k}$ имеется ровно один дивизор $F$, инвариантный относительно группы $K^{*} \times S L_{2},-$ это слой морфизма факторизации для группы $S L_{2}$, содержащий неподвижную точку. Функция $z$ является $S L_{2}$-инвариантной и собственной веса $\epsilon$ для тора $K^{*}$.

Пусть $q$ - порядок нуля функции $z$ на дивизоре $F$. Функция $\frac{a^{2 k}}{\left(b^{2}-4 a c\right)^{k}}$ является $K^{*}$-инвариантной и полуинвариантной веса $4 k \lambda$ для $S L_{2}$. На дивизоре $F$ эта функция имеет полюс порядка $q n$. Итак, дивизору $F$ соответствует нормирование, заданное в наших обозначениях координатами $(-q n / k, q)$. Соответствующий конус задается парой $(n, k)$.

Многообразие $X_{n}^{k}$ получается из многообразия $X^{k}$ переходом к расслоенному произведению относительно морфизма факторов $z \rightarrow z^{n}$. Поэтому число $k$ отвечает за порядок стабилизатора точки из незамкнутой орбиты особого слоя многообразия $X_{n}^{k}$, а число $n$ есть своего рода “индекс вращения" многообразия вокруг особого слоя. Нечто подобное возникает в теории действий компактных групп на трехмерных многообразиях. А именно, в классификации Реймонда эффективных и гладких действий окружности на гладких замкнутых связных 3-многообразиях из таких же соображений вводятся ориентированные инварианты Зейферта, см., например, [15]. 


\section{6. Классификация $(S, T)$-многообразий}

Аналогично $(S, N)$-многообразиям классифицируются $(S, T)$-многообразия бирационально тривиального типа. Единственное изменение, которое необходимо внести, - это в строке (1) заменить $V_{4 r_{1}}$ на $V_{2 r_{1}}$. Теорема 3 справедлива и в этом случае.

Отсюда следует, что на бирационально тривиальных $(S, T)$-многообразиях не может быть орбиты типа $S L_{2} / N$. Это можно получить и из теории этального слайса - если бы $X^{T}$ было приводимо, то и слайс в окрестности замкнутой орбиты типа $S L_{2} / N$ был бы приводим и связен, и потому не нормален, а нормальность точки на слайсе эквивалентна нормальности точки на многообразии.

Однородное пространство $S L_{2} / T$ допускает ровно один нетривиальный $S L_{2}$-эквивариантный автоморфизм, который согласно леммы 3.2 можно продолжить на всякое $(S, T)$-многообразие. После факторизации по такому действию группы $\mathbb{Z}_{2}$ получается $(S, N)$-многообразие.

ПРЕДЛОЖЕНИЕ 7. Всякое $(S, N)$-многообразие может бъть двулистно накрыто указанным выше способом ровно одним $(S, T)$-многообразием бирационально тривиального типа. При этом отметка $\left(k_{i}, n_{i}\right)$ на $(S, N)$-многообразии перейдет в отметку $\left(2 k_{i}, n_{i}\right)$ при нечетном $n_{i}$ и в отметку $\left(k_{i}, n_{i} / 2\right)$ при четном $n_{i}$. В первом случае неподвижная точка является изолированной точкой ветвления, а во втором случае имеется дивизор ветвления.

Доказательство вытекает из того факта, что действие $\mathbb{Z}_{2}$ коммутирует с операциями перехода к расслоенному произведению и склейки.

Рассмотрим бирационально нетривиальное $(S, T)$-многообразие $X$. Условие нетривиальности эквивалентно неприводимости кривой $X^{T}$. Согласно предложению 3 данное многообразие $X$ можно двулистно накрыть бирационально тривиальным $(S, T)$-многообразием $\widetilde{X}$, причем накрьвающий морфизм будет морфизмом факторизации по группе $\mathbb{Z}_{2}$ (двулистное накрытие всегда является накрытием Галуа). Такое накрытие определено однозначно, так как алгебра регулярных функций на $\widetilde{X}$ совпадает с целым замыкание алгебры $K[X]$ в канонически определенном расширении степени 2 поля $k(X)$.

ПримеР. Трехмерное пространство присоединенного представления группы $S L_{2}$ является бирационально нетривиальным $(S, T)$-многообразием. Двулистно накрывающее его бирационально тривиальное $(S, T)$-многообразие есть гиперповерхность в четырехмерном пространстве, заданная уравнением $z^{2}=b^{2}-4 a c$. В терминах нашей классификации бирационально тривиальных $(S, T)$-многообразий этой гиперповерхности отвечает прямая с единственной отметкой $(1,1)$ в точке 0.

$S L_{2}$-эквивариантное действие групшы $\mathbb{Z}_{2}$ на $\widetilde{X}$ индуцирует нетождественное действие $\mathbb{Z}_{2}$ на факторе $\widetilde{X} / / S L_{2}=C$. Из классификации бирационально тривиальных $(S, T)$-многообразий вытекает, что для того чтобы задать бирационально тривиальное $(S, T)$-многообразие с требуемьм действием группы $\mathbb{Z}_{2}$ необходимо и достаточно задать неприводимую гладкую афффинную кривую с нетождественным действием группы $\mathbb{Z}_{2}$, а также расставить на ней отметки с единственным ограничением - точки, лежащие на одной $\mathbb{Z}_{2}$-орбите должны быть либо обе не отмеченными, либо отмечены одной и той же парой чисел (в частности, на отметки 
в $\mathbb{Z}_{2}$-неподвижных точках никаких ограничений не накладывается). Будем называть такую систему отметок $\mathbb{Z}_{2}$-инвариантной.

Теорема 4. Бирационально нетривиальные $(S, T)$-многообразия находятся во взаимно однозначном соответствии со следующим набором данных:

1) $C$ - гладкая неприводимая аффинная кривая с заданным на ней нетождественныц действием группь $\mathbb{Z}_{2}$;

2) $\mathbb{Z}_{2}$-инвариантная система отметок на кривой $C$ (возможно, пустая).

ЗАмечАния. 1) Неотмеченные $\mathbb{Z}_{2}$-неподвижные точки кривой $C$ отвечают слоям типа $S L_{2} / N$ на многообразии $X$.

2 ) Для бирационально нетривиального $(S, T)$-многообразия $X$ неприводимая кривая $X^{T}$ не обязательно является гладкой. В частности, она заведомо особа, если отметки на кривой $C$ расставлены не только в $\mathbb{Z}_{2}$-неподвижных точках.

\section{Список литературы}

1. Винберг Э. Б. Сложность действий редуктивных групп // Функ. анализ и его прил. 1986. T. 20. № 1. C. 1-13.

2. Аржанщев И. В. О действиях сложности один групाы $S L_{2}$ // Изв. РАН. Сер. матем. 1997 (в печати).

3. Крафт X. Геометрические методы в теории инвариантов. М.: Мир, 1987.

4. Попов В. Л. Квазиоднородные афффинные алгебраические многообразия группы $S L(2)$ // Изв. АН СССР. Сер. матем. 1973. Т. 37. № 4. С. 792-832.

5. Kempf G., Knudson F., Mumford D., Saint-Donat B. Toroidal embeddings // Lecture Notes in Math. 1973. V. 337.

6. Тимашев Д. А. G-многообразия сложности один // УМН. 1996. Т. 51. №3. С. 213-214.

7. Винберг Э.Б., Попов В.Л. Теория инвариантов // Итоги науки и техники. Совр. пробл. матем. Фундам. направления. Т. 55. М.: ВИНИТИ, 1989. С. 137-314.

8. Хамфри Дж. Линейные алгебраические группы. М.: Наука, 1980.

9. Хартсхорн Р. Алгебраическая геометрия. М.: Мир, 1981.

10. Шафаревич И. Р. Основы алгебраической геометрии. М.: Наука, 1988.

11. Попов В. Л. Стягивание действий редуктивных алгебраических групा // Матем. сб. 1986. T. 130 (172). №3 (7). C. $310-334$.

12. Knop F. The Luna-Vust Theory of Spherical Embeddings // Proc. of the Hyderabad Conf. on Algebraic Groups. Madras: Manoj Prakashan, 1991. P. 225-249.

13. Knop F. The Asymptotic behavior of the invariant collective motion // Invent. Math. 1994. V. 116. P. 309-328.

14. Bialynicki-Birula A., Swiecicka J. Good Quotients for Actions of $S L(2) / /$ Bull. Polish Acad. Sci. Math. 1988. V. 36. P. 375-381.

15. Orlik P. Seifert manifolds // Lecture Notes in Math. 1972. V. 291.

Московский государственньй университет им. М.В. Ломоносова 